\title{
Fine Structure of Highly Charged Excitons in Semiconductor Quantum Dots
}

\author{
B. Urbaszek, ${ }^{1}$ R. J. Warburton, ${ }^{1}$ K. Karrai, ${ }^{2}$ B. D. Gerardot, ${ }^{3}$ P. M. Petroff, ${ }^{3}$ and J. M. Garcia ${ }^{4}$ \\ ${ }^{1}$ Department of Physics, Heriot Watt University, Edinburgh EH14 4AS, United Kingdom \\ ${ }^{2}$ Center for NanoScience and Sektion Physik, Ludwig-Maximilians-Universität, \\ Geschwister-Scholl-Platz 1, 80539 München, Germany \\ ${ }^{3}$ Materials Department and QUEST, University of California, Santa Barbara, California 93106, USA \\ ${ }^{4}$ Instituto de Microelectrónica de Madrid, CNM-CSIC Isaac Newton 8, PTM, 28760 Tres Cantos, Madrid, Spain
}

(Received 29 October 2002; published 19 June 2003)

\begin{abstract}
An exciton in a symmetric semiconductor quantum dot has two possible states, one dark and one bright, split in energy by the electron-hole exchange interaction. We demonstrate that for a doubly charged exciton, there are also two states split by the electron-hole exchange, but both states are now bright. We also uncover a fine structure in the emission from the triply charged exciton. By measuring these splittings, and also those from the singly charged and doubly charged biexcitons, all on the same quantum dot, we show how the various electron-hole exchange energies can be measured without having to break the symmetry of the dot.
\end{abstract}

DOI: 10.1103/PhysRevLett.90.247403

Semiconductor quantum dots (QDs) are artificial atoms whose properties can be individually measured in their solid-state environment [1]. To manipulate the charge and spin of an exciton confined to a QD, it is necessary to understand the Coulomb interactions between electrons and holes. In particular, for applications of QDs as single photon emitters, the exchange interaction between electrons and holes is crucial as this can determine the polarization and entanglement of the emitted photons [2]. For nonisotropic QDs, the degeneracy of the excitonic ground state is lifted by the electron-hole (EH) exchange interaction, resulting in two distinguishable pathways for the biexciton decay, prohibiting the biexciton as a source of entangled photons. While the EH exchange interactions in semiconductor QDs are typically less than a meV, they can be much larger in semiconductor nanocrystals [3] or polymers [4]. In all these systems, it is difficult to obtain a complete picture of the EH exchange interaction because the exciton is split into bright $(m= \pm 1)$ and dark $(m=$ \pm 2 ) states. Only the bright states can emit a photon as a consequence of the selection rule on the spin angular momentum, $m$. The EH exchange interaction of a QD exciton has typically been measured by breaking the symmetry, either with a strong magnetic field or with a highly anisotropic strain field, mixing the bright and dark states. From these special cases, general conclusions have been extrapolated [5].

A new approach to probing the EH exchange interaction is to measure the emission from more complicated exciton complexes. Akimov et al. have shown that in the emission from the charged biexciton, the final state is split by the EH exchange interaction, the splitting measuring directly a particular EH exchange energy [6]. In this Letter we show how a complete picture of the EH exchange interaction can be gleaned, even for isotropic dots at zero magnetic field, by measuring the emission characteristics of various charged excitons. We show that
PACS numbers: 78.55.Cr, 71.70.Gm, 73.21.La, 78.67.Hc

for a doubly charged exciton, the EH exchange interaction splits the exciton into two states, but, unlike the case of a neutral exciton, both states are now bright. We explain how the exchange interactions between the hole ground state and the electron ground and excited states can all be determined by combining the results on the doubly and triply charged excitons with those on charged biexcitons. As we show, emission from all these excitons can be measured on the same QD.

We employ QDs embedded in a field-effect structure between a highly doped GaAs layer and a surface gate electrode that allows us to control precisely the charge of the exciton [7]. In these experiments, a hole is generated with optical excitation. Over large regions of bias voltage, the excitonic charge is constant, and there are abrupt steps in emission energy whenever an additional electron is added to the dot [8]. The dots have either an ensemble photoluminescence (PL) centered at $1.3 \mathrm{eV}$ (sample $A$ ) or at $1.1 \mathrm{eV}$ (sample $B$ ) depending on the growth. We have performed PL spectroscopy of individual QDs as a function of gate voltage $V_{g}$ at $4.2 \mathrm{~K}$ using a confocal microscope. The PL is excited with an $850 \mathrm{~nm}$ laser diode, dispersed with a grating spectrometer and detected in linear polarization with a Si charge-coupled device camera. The spectral resolution (precision) is $0.05(0.02) \mathrm{meV}$. We can determine absolute (relative) single dot PL intensities to an accuracy of about $15 \%(20 \%)$. We find that the dots are close to isotropic in the $(x, y)$ plane $(z$ is the growth direction). The anisotropic EH exchange splitting, measured for the neutral exciton in a transmission experiment with a narrow linewidth laser to be $0.02 \pm$ $0.01 \mathrm{meV}$ [9], is much smaller than the isotropic $\mathrm{EH}$ exchange interaction which we determine here to be $\sim 0.6 \mathrm{meV}$. To within our experimental uncertainty in the relative intensity we find no dependence of the PL from the triply charged exciton and from the singly charged biexciton on the direction of the polarization. 
This is consistent with the small value of the anisotropic $\mathrm{EH}$ exchange relative to the isotropic $\mathrm{EH}$ exchange, as larger anisotropy gives rise to elliptically polarized charged biexciton emission [6].

At low laser power, for each individual dot, we can identify the neutral exciton, $X^{0}$, the charged exciton, $X^{1-}$, the doubly charged exciton, $X^{2-}$, and the triply charged exciton, $X^{3-}$, by the large jump in PL energy on going from $X^{0}$ to $X^{1-}$, and the characteristic splittings of $X^{2-}$ and $X^{3-}$, as shown in Fig. 1 . The $X^{2-}$ is split into two lines because there are two possible final states after photon emission, a triplet and a singlet, which are split by the electron-electron (EE) exchange interaction [8]. The splitting is $2 X_{s p}^{e e}$, where $X_{s p}^{e e}$ is the exchange energy between an electron in the ground $s$ state and an electron in the excited $p$ state, as shown diagrammatically in Fig. 2. In previous experiments, the splitting was typically $4 \mathrm{meV}$ $[8,10]$. By turning to sample $B$, we find that the splitting increases to $7 \mathrm{meV}$ as a result of the stronger confinement. This enhancement enables us to resolve a new splitting: the emission into the triplet is not a single line, but a doublet. Examples are shown in Figs. 2 and 3. The $X^{0}$ and $X^{1-}$ emission consist of single lines to within our spectral resolution. We attribute the new splitting of the $X^{2-}$ to the $\mathrm{EH}$ exchange interaction. An elegant feature is that the $X^{2-}$ emission gives an immediate measure of the EE and EH exchange interactions. The EH exchange is an order of magnitude smaller than the EE exchange because, while two electrons are indistinguishable, an electron can be distinguished from a hole by the Bloch part of the wave function.

To explain the new splitting of the $X^{2-}$ triplet emission, we consider disk-shaped quantum dots in the $(x, y)$ plane. For the electrons, we allow for the possibility that the two

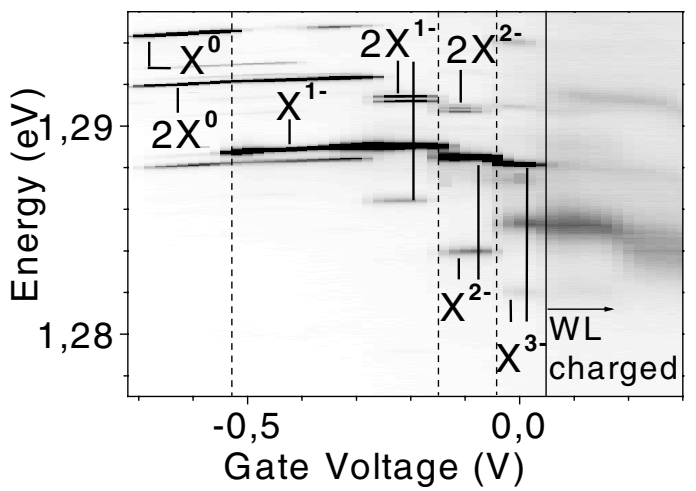

FIG. 1. Gray-scale plot of the photoluminescence versus gate voltage for a single dot from sample $A$ at $4.2 \mathrm{~K}$. White corresponds to 0 counts, black to 4000 counts on the detector. The excitons are labeled with $X$ standing for exciton, $2 X$ for biexciton, with excess charge as the suffix. Dotted lines mark charging events. The unlabeled emission at $1.288 \mathrm{eV}$ is related to $2 X^{0}$, but its exact origin is presently unknown. The power of the $850 \mathrm{~nm}$ laser was chosen so that the biexciton features are clearly visible. Above a voltage of about $0.05 \mathrm{~V}$, the wetting layer (WL) loads with electrons. $p$ states are not exactly degenerate. We label the two states $p_{x}$ and $p_{y}$ with $p_{x}$ having a lower energy than $p_{y}$. The hole is taken to have purely heavy hole character because the light hole states are energetically distant due to strain. We assume initially that the dot wave functions do not change appreciably on occupying the states; as we shall see, the experiments allow us to examine this assumption. The exchange interaction for $C_{2 v}$ symmetry can be expressed by the effective spin Hamiltonian [11]:

$$
\hat{H}_{e x}=2 \Delta J_{z} S_{z}+\Delta_{1}\left(J_{x} S_{x}-J_{y} S_{y}\right)+\Delta_{2}\left(J_{x} S_{x}+J_{y} S_{y}\right),
$$

where $S_{z}\left(J_{z}\right)$ is the $z$ component of the total electron spin $S$ (hole pseudospin $J$ ). $J_{z}$ takes values $\frac{1}{2}$ and $-\frac{1}{2}$ corresponding to hole spin $-\frac{3}{2}$ and $\frac{3}{2}$, respectively.

The first term in the Hamiltonian is the isotropic EH exchange and splits the neutral exciton, $X^{0}$, into two states, one bright and one dark. The second and third terms introduce an anisotropy, splitting both bright and dark states into doublets such that the two bright states couple to photons having orthogonal linear polarization, but they do not admix the bright and dark states. This gives rise to two closely spaced lines in the $X^{0}$ absorption [9], but they are too close together for us to resolve in the PL experiment. We do not observe any splittings arising from $\Delta_{1,2}$ with our present PL setup, implying that for our dots $\Delta \gg \Delta_{1,2}$ and $\Delta_{1,2} \leq 0.05 \mathrm{meV}$, our experimental resolution. For the singly charged exciton, $X^{1-}$, the electron $s$ state is occupied with a spin up and a spin down electron such that the total spin is zero. For this reason, the EH exchange is zero. This is confirmed in the experiment: as for $X^{0}$, we see a single, sharp line for $X^{1-}$, as shown in Fig. 3. However, for the doubly charged exciton, $X^{2-}$, the EH exchange reappears because the electron

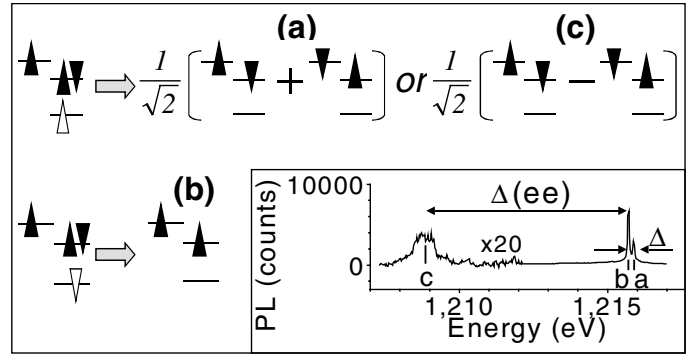

FIG. 2. Main figure: level diagrams for the initial and final states of a doubly charged exciton $X^{2-}$ in a QD. The states shown are the hole $s$ state, the electron $s$ state and the electron $p_{x}$ state. Electrons (holes) are shown with solid (open) arrows; an up-arrow (down-arrow) denotes spin up (spin down). The pseudospin is used for the hole. The two initial states differ in energy by the electron-hole exchange interaction $\Delta$. There are three possible final states: (a) $S, S_{z}=1,0$; (b) 1,1 ; and (c) 0,0 . State (c) is split from (a) and (b) by electron-electron exchange, $\Delta(e e)$. The inset shows a typical $X^{2-}$ emission spectrum from a dot from sample $B$. The transitions are labeled with (a), (b), (c) to denote the final state, as in the level diagrams. 


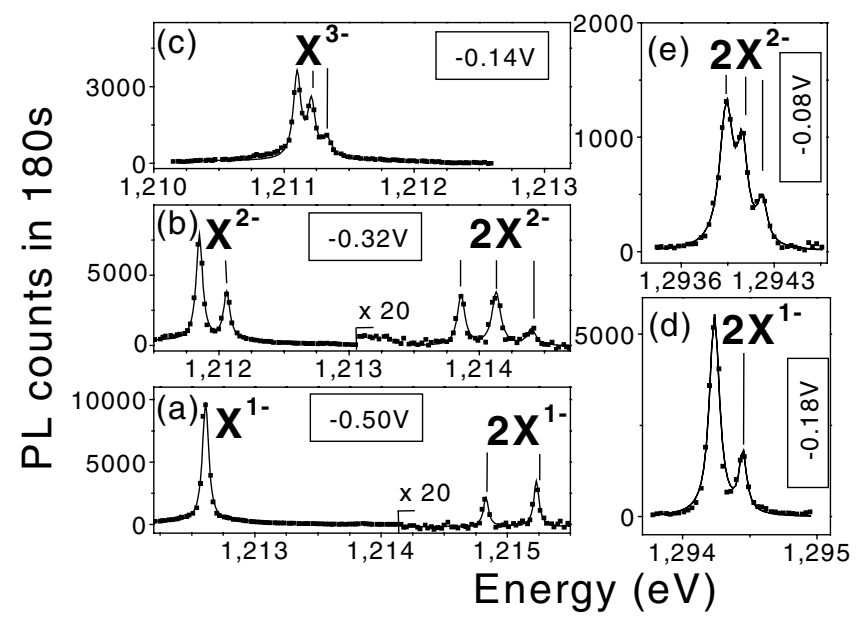

FIG. 3. (a) $X^{1-}$, (b) $X^{2-}$, (c) $X^{3-}$ emission spectra for dot I from sample $B$ at $4.2 \mathrm{~K}$. The voltages applied to generate the various excitons are labeled in each case. Also shown are (d) $2 X^{1-}$ and (e) $2 X^{2-}$ emission spectra for a dot from sample $A$ at $4.2 \mathrm{~K}$. Symbols are experimental data, and lines are fits to Lorentzian line shapes.

spin $S=\frac{1}{2}$. The initial configurations for $S_{z}=+\frac{1}{2}$ are sketched in Fig. 2. If the hole pseudospin is $J_{z}=-\frac{1}{2}\left(+\frac{1}{2}\right)$, the EH exchange decreases (increases) the initial state energy, the splitting between the two possibilities being exactly $\Delta$. The important point is that both initial states are bright because in both cases the hole can find a partner for recombination in the doubly occupied electron $s$ state. As a result the emission into the triplet state should have two closely spaced lines, exactly as in the experiment. A related result is that emission into the singlet state is not split by the $\mathrm{EH}$ exchange interaction. By considering the different final state wave functions for $S, S_{z}=1,1$ and $S, S_{z}=1,0$, as sketched in Fig. 2, the fine structure lines are expected to have an intensity ratio of 2:1, assuming that the population of the initial states is equally likely. Averaging over tens of dots from sample $B$, we find experimentally that the intensity ratio is $2: 0.8 \pm 0.4$, in excellent agreement with the calculation. In general, the value of $\Delta$ depends on the particular exciton. For $X^{2-}$ $\Delta\left(X^{2-}\right)=X_{p_{x} s}^{e h}$; for dot I, $X_{p_{x} s}^{e h}=0.21 \mathrm{meV}$. The parameters for several dots are listed in Table I.

Confirmation of this analysis comes from the triply charged exciton, $X^{3-}$. For $X^{3-}$, we observe, as for $X^{2-}$, a splitting of the PL due to EE exchange (see Fig. 1). This proves that the two $p$ electrons in the $X^{3-}$ ground state have parallel spins $(S=1)$, with one electron in the $p_{x}$ state, the other in the $p_{y}$ state. (If the energy separation between $p_{x}$ and $p_{y}$ is larger than the exchange energy $X_{p_{x}}^{e e} p_{y}$, the $p_{x}$ orbital is doubly occupied such that $S=0$. In the case of an $S=0 X^{3-}$, the PL consists of a single line between the singlet and triplet states of the $X^{2-}$ emission [10].) The final states have $S=\frac{3}{2}$ or $S=\frac{1}{2}$, split by $3 X_{s p}^{e e}$ [8]. The $X^{3-}$ initial states have $S_{z}=1,0,-1$, leading us to predict the existence of three equidistant lines in the emission into the triplet final state. This is
TABLE I. Measured exchange energies for three different dots from sample $B ; \Delta^{*}\left(2 X^{2-}\right)$ is the calculated and $\Delta\left(2 X^{2-}\right)$ is the measured splitting, all values in $\mathrm{meV}$.

\begin{tabular}{cccrcc}
\hline \hline Dot no. & $X_{s s}^{e h}$ & \multicolumn{1}{c}{$X_{p_{x} s}^{e h}$} & \multicolumn{1}{c}{$X_{p_{y} s}^{e h}$} & $\Delta^{*}\left(2 X^{2-}\right)$ & $\Delta\left(2 X^{2-}\right)$ \\
\hline I & 0.56 & 0.20 & -0.02 & 0.25 & 0.25 \\
II & 0.63 & 0.21 & 0.01 & 0.28 & 0.26 \\
III & 0.57 & 0.21 & 0.01 & 0.26 & 0.27 \\
Abs. error & \pm 0.05 & \pm 0.02 & \pm 0.05 & \pm 0.05 & \pm 0.02 \\
\hline \hline
\end{tabular}

exactly what we observe, as shown in Fig. 3. Furthermore, by taking into account the configuration mixing in the final states, we predict an intensity ratio of 3:2:1 for the fine structure lines, again assuming equally likely initial state occupation. Experimentally, we measure 3:2.0 \pm 0.1:0.9 \pm 0.4 , in extremely good agreement with our prediction. (We note that emission into the $X^{3-} S=\frac{1}{2}$ final state should be split into two lines by the EH exchange interaction, but because the singlet final state has a linewidth of about $0.5 \mathrm{meV}$ due to fast relaxation, the EH exchange effects cannot be discerned for this transition.) For $X^{3-}, \Delta\left(X^{3-}\right)=\frac{1}{2}\left(X_{p_{x} s}^{e h}+X_{p_{y} s}^{e h}\right)$. Given that $X_{p_{x} s}^{e h}$ is known from the $X^{2-}$ splitting, we find for dot I that $X_{p_{y} s}^{e h} \simeq 0$. This surprising result is confirmed on other dots (see Table I).

For applications involving neutral excitons, the most important parameter is the $\mathrm{EH}$ exchange between an $s$ electron and an $s$ hole. We can determine this energy by turning to charged biexcitons. For biexcitons, there is no EH exchange interaction in the initial state because the hole spin is zero. However, EH exchange can split the final states as one hole is left behind after recombination [6]. For instance, for the singly charged biexciton, $2 X^{1-}$, there are two final states after photon emission, $S=1$ and $S=0$. For $S=1$, the $S_{z}=1$ state is no longer degenerate with the $S_{z}=0$ state because of the presence of the hole, leading to two closely separated lines in the emission, separated by $\Delta\left(2 X^{1-}\right)=\frac{1}{2}\left(X_{s s}^{e h}+X_{p_{x} s}^{e h}\right)$. The final state of the $2 X^{1-}$ is therefore the same as the initial state of the so-called hot trion [6,12]. Experimentally, we can access the charged biexcitons by increasing the laser power so that a particular dot is occasionally occupied by two holes (Fig. 1). The neutral biexciton, $2 X^{0}$, is identified by its superlinear dependence on laser power, and also by the fact that it exists over a large range of $V_{g}$, just as for $X^{1-}$. (For both $X^{1-}$ and $2 X^{0}$ the Coulomb blockade is pronounced because an additional electron has to overcome both the Coulomb energy barrier and the quantization energy barrier.) At a particular $V_{g}$, the biexciton state changes from $2 X^{0}$ to $2 X^{1-}$, as revealed by the singlet-triplet splitting in the PL. Example $2 X^{1-}$ emission spectra are shown in Fig. 3. Exactly as expected, the fine structure consists of two closely separated lines. A clear result is that the splitting is larger than for $X^{2-}$ implying that $X_{s s}^{e h}>X_{p_{x} s}^{e h}$. $X_{s s}^{e h}$ is typically $0.6 \mathrm{meV}$ for dots in sample $B$ (see Table I). 
The doubly charged biexciton, $2 X^{2-}$, shows three peaks in its fine structure, as shown in Fig. 3. This confirms our analysis in two ways. First, this is exactly as expected from the spin Hamiltonian. Again, a 3:2:1 intensity ratio is expected from the configuration admixture; we measure 3:2.3 \pm 1.0:0.8 \pm 0.2. Second, for $2 X^{2-}$, the fine structure lines are separated by an energy $\Delta\left(2 X^{2-}\right)=\frac{1}{3}\left(X_{s s}^{e h}+X_{p_{x} s}^{e h}+X_{p_{y} s}^{e h}\right)$. This gives us a means to check our EH exchange energies because the system is overdetermined: $X_{s s}^{e h}, X_{p_{x} s}^{e h}$, and $X_{p_{y} s}^{e h}$ are known from the other fine structure splittings. We calculate $\Delta\left(2 X^{2-}\right)$ and compare it to the measured value in Table I. For all our dots, we achieve perfect agreement. There are larger fluctuations from dot to dot in the relative intensities for the charged biexcitons than for the excitons, probably due to dot-dependent configuration mixing in the closely spaced valence states which plays a role for the biexcitons but not for the excitons.

In the short-range approximation, the EH exchange interaction is proportional to the probability that the electron and hole are located in the same unit cell [5]. From the form of the wave functions, this probability is less for a $p$ electron and $s$ hole than for an $s$ electron and an $s$ hole, giving a qualitative explanation of our result that $X_{p_{x} s}^{e h}<X_{s s}^{e h}$. Quantitatively, taking two-dimensional harmonic oscillator wave functions for the electrons and holes [13], we find that $X_{s s}^{e h} \propto 1 /\left(L_{e}^{2}+L_{h}^{2}\right)$, whereas $X_{p s}^{e h} \propto$ $L_{h}^{2} /\left(L_{e}^{2}+L_{h}^{2}\right)^{2}$, where $L_{e}\left(L_{h}\right)$ is the confinement length for the electron (hole). This result gives reasonable agreement with the experiments using $L_{e}=5.2 \mathrm{~nm}$ and $L_{h}=$ $3.5 \mathrm{~nm}$ [13]. The small value of $X_{p_{y} s}^{e h}$ is an unexpected result. It seems unlikely that it arises from a highly anisotropic dot shape for three reasons. First, for our dots $\Delta \gg \Delta_{1,2}$, strong evidence for weak anisotropy. [For $X^{3-}$ the anisotropy-related terms in Eq. (1) increase the fine structure splitting only by an amount second order in $\Delta_{1,2} / \Delta$, and so do not influence our determination of $X_{p_{y} s}^{e h}$.] Second, the form of the $X^{3-}$ PL is itself evidence for a symmetric dot. We observe in the $X^{3-}$ PL a large splitting related to EE exchange which, as explained above, allows us to deduce that the $p_{x}$ and $p_{y}$ states are split by no more than $X_{p_{x} p_{y}}^{e e} . X_{p_{x} p_{y}}^{e e}$ is typically only a few $\mathrm{meV}$ and is therefore small compared to the splitting between the $s$ and $p$ states, typically $30 \mathrm{meV}$. Third, a highly distorted hole state could explain the small value of $X_{p_{y} s}^{e h}$. However, a distorted hole wave function would imply a much reduced overlap between the $s$ hole and $s$ electron wave functions. We find no evidence for this: the radiative lifetime of these dots is $\sim 800 \mathrm{ps}$ which is typical for strongly confined InAs dots in GaAs and corresponds to an almost complete EH overlap. At present, a full explanation of the fact that $X_{p_{y} s}^{e h} \simeq 0$ is missing. It might be significant that we can measure $X_{p_{y} s}^{e h}$ only when both $p_{x}$ and $p_{y}$ states are occupied. Possibly occupation of the states increases the extent of the electron $p$ wave function such that the overlap with the tightly confined hole is reduced. In this picture, our results can be described by saying that $X_{p s}^{e h}$ takes the value $X_{p_{x} s}^{e h}$ when the $p$ state is singly occupied $\left(X^{2-}\right.$ and $\left.2 X^{1-}\right)$, reducing to $\sim 0.5 X_{p_{x} s}^{e h}$ when the $p$ state is doubly occupied $\left(X^{3-}\right.$ and $\left.2 X^{2-}\right)$. Two observations support this idea. First, for the dots described here, $X^{3-}$ is the most highly charged exciton we observe, suggesting that the energy of the $X^{3-}$ initial state lies close to the top of the confining potential where a softening of the potential can be expected. Second, $X_{s p}^{e e}$ determined from $X^{3-}$ is smaller than that determined from $X^{2-}$, also suggesting a softening of the potential. However, on going from $X^{2-}$ to $X^{3-}, X_{p s}^{e h}$ decreases more significantly than $X_{s p}^{e e}$; while for $L_{e} \gg L_{h}$, $X_{p s}^{e h}$ scales as $1 / L_{e}^{4}$, making $X_{p s}^{e h}$ very sensitive to $L_{e}$, it is at this stage unclear if this alone accounts for the experimental results.

To conclude, we demonstrate that the emission from doubly and triply charged excitons in a QD is split by the EH exchange interaction. By combining this information with that from the fine structure splittings of the singly and doubly charged biexcitons, we show how we can deduce a complete picture of the EH exchange interaction. This represents a novel method of determining the exciton fine structure in a semiconductor nanostructure without having to break the symmetry. Furthermore, we find that there are no dark states for highly charged excitons, and this could be exploited for more efficient emission from photonic materials. EH exchange effects have to be taken into account when manipulating exciton spins in QDs and also for the generation of entangled photons from biexciton decay, which remains a very promising possibility. We hope that our results stimulate new attempts to achieve this.

This work was financially supported by EPSRC (U.K.) and DFG (SFB348). We thank K. V. Kavokin for stimulating discussions.

[1] A. Zrenner, J. Chem. Phys. 112, 7790 (2000).

[2] C. Santori et al., Phys. Rev. B 66, 045308 (2002).

[3] A. L. Efros et al., Phys. Rev. B 54, 4843 (1996).

[4] R. H. Friend et al., Nature (London) 397, 121 (1999).

[5] M. Bayer et al., Phys. Rev. B 65, 195315 (2002), and references therein.

[6] I. A. Akimov et al., Appl. Phys. Lett. 81, 4730 (2002).

[7] H. Drexler et al., Phys. Rev. Lett. 73, 2252 (1994).

[8] R. J. Warburton et al., Nature (London) 405, 926 (2000).

[9] K. Karrai et al. (unpublished).

[10] J. J. Finley et al., Phys. Rev. B 63, 161305 (2001).

[11] E. L. Ivchenko and G. E. Pikus, Superlattices and Other Heterostructures (Springer-Verlag, Berlin, 1997).

[12] I. E. Kozin et al., Phys. Rev. B 65, 241312 (2002).

[13] R. J. Warburton et al., Phys. Rev. B 58, 16221 (1998). 